Simeonov, Plamen L.:

On using nomadic services for distributed intelligence

Zuerst erschienen in:

Microprocessors and Microsystems 24 (2000), Nr. 6, S. 291-297 DOI: 10.1016/S0141-9331(00)00089-2 und in:

Proceedings // Eight International Conference on Computer Communications and Networks : 11 - 13 October 1999, Boston, Massachusetts / ed. by Sudhir Dixit ... [ICCCN 8, 1999]. - Piscataway, NJ : IEEE, 1999. - ISBN 0-7803-5794-9, S. 228-231

DOI: $10.1109 / \mathrm{ICCCN} .1999 .805523$ 


\title{
On using nomadic services for distributed intelligence
}

\author{
P.L. Simeonov* \\ Siemens AG, Siemensdamm 50, D-13623 Berlin, Germany
}

\begin{abstract}
This paper presents a next generation integrated IN architecture among Flexible Intelligent Network Elements (FINE) based on the multimedia multimode (client, server and agent) IN Service Node concept (Proc. ICCE '97, Cannes (1997) 77), to perform a crafty Unified Media Communications Service (UMCS) across heterogeneous networks. The FINE architecture represents a network of configurable interworking elements allowing user access to a Unified Message Store (UMS) and Universal Communications Channel (UCC) via traditional PSTN/ISDN/PLMN equipment such as telephones, pagers and fax machines on the one side, and networked computers and mobile terminals equipped with mail readers and Web browsers on the other side, to enable both on-line and off-line interactions. A virtual cluster of FINEs within an integrated personal communications network is dynamically configured in a distributed or centralised manner according to user profile requirements, network size and performance to provide artful messaging, telephony and $I^{3}$ E services (Intelligent Inter-, Intra- and Extranet) in a changing environment.

The FINE consists of a Channel Matrix Switch (CMS), several Resource Platforms (RP) containing Media Conversion Processors (MCP) and Channel Managers (CM) to perform the media translation and routing in the required interchange formats, an Internet Gateway (IG) to hold the subscribers' mailboxes and provide the internet connectivity, and a FINE Controller (FINEC) to realise the FINE Service Logic (FSL) and manage the FINEs.

Each FINEC can be deployed in some of the three modes — independent (server), dependent (client) and autonomous (agent) — with respect to the user/network configuration, thus allowing a dynamically configurable (per user/ per node/ per service) centralised or distributed service architecture.

The FINE itself is organised along with other nodes by a Network Operation Support Environment (NOSE), a service oriented and TMN compliant Operation, Administration \& Maintenance Centre.

Service logic can be transferred, installed and mounted on demand among the FINE Controllers and the terminal equipment to provide optimal QoS. (C) 2000 Elsevier Science B.V. All rights reserved.
\end{abstract}

Keywords: Intelligent networks; Service architecture; Network convergence; Nomadic service Logic

Whatever is flexible and flowing will tend to grow... (Dao De Jing)

\section{Introduction}

The core of the proposed new IN architecture is FINE, the result of a joint project initiative, @INGate, between Siemens AG and the Technical University Berlin, [1]. The goal of this project was to design an InterWorking Unit (IWU) as a mediation device between existing circuit and packet switching networks, by using traditional ISDN/ PSTN/PLMN equipment (TE2) and Internet Terminals (IT), cf. Fig. 1, [2].

\footnotetext{
* Tel.: +49-30-386-24346; fax: +49-30-386-31110.

E-mail address: plamen.simeonov@icn.siemens.de (P.L. Simeonov).
}

Our prototype hosts a generic Unified Media Communication Service (UMCS) composed of multimedia messaging (store-and-forward), telephony and agent-based network computing to provide integrated information services between telephony and data networks.

\section{The reference model}

The @INGate reference model contains the following network elements (Fig. 2).

- Flexible Intelligent Network Element (FINE).

- FINE Data Server (FineDS).

- Network Operation Support Environment (NOSE).

- WWW server.

- Firewall.

The Network-Bridge FINE architecture represents 


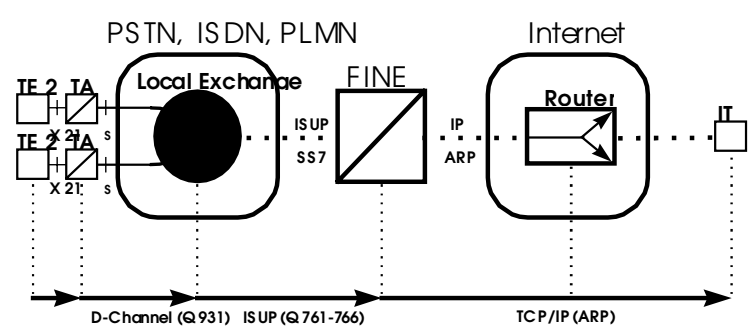

Fig. 1. The Network-Bridge FINE as an InterWorking Unit.

InterWorking Unit between PSTN/ ISDN/ PLMN and the internet. It is based on the Service Node concept in Intelligent Networks that was extended by cunning media treatment and routing techniques along with enhanced SRF, FSL and scheduling mechanisms.

The FINE consists of:

- a FINE Controller (FINEC);

- one or more Resource Platforms (RPs);

- a Channel Matrix Switch (CMS);

- a Data Packet Switch (DPS);

- Media Conversion Processors (MCPs);

- an Internet Gateway (IG) with

- a Unified Media Store/Bus (UMS/B), and

- a Personal IN Gateway Area (PINGA).

All FINE components except the FINE Controller, which carries multiple service logic, are optional and configurable to allow a multimode functionality of each single element.

The FINE Data Server (FineDS) holds all service and subscriber relevant information such as service configuration and user profile records.

The Network Operation Support Environment allows access to all FINE components (and of the neighbour FINEs) via remote control sessions to UNIX/WEB hosts (FINECs) or via SNMP-based management.

The WWW server supports WWW-based management of all FINE components in addition to the TMN-conform NOSE workstation, as well as Customer Service Control (CSC) such as configuration of service specific parameters

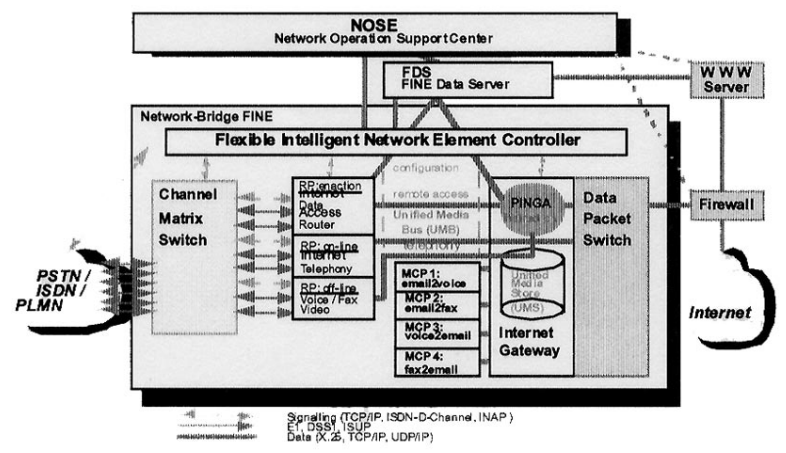

Fig. 2. The @INGate Reference Model.

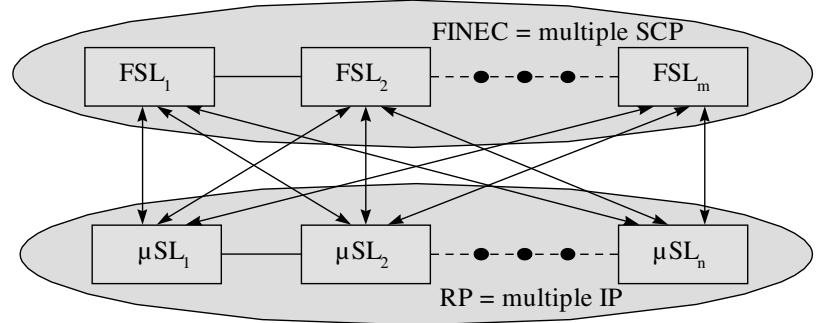

Fig. 3. Distribution of service logic between SCP and IP.

through the internet by using security-enhanced web browsers.

The Firewall computer connects the FINE to the internet.

\section{The FINE architecture}

This section reviews the FINE components in detail.

\subsection{A FINE Controller}

The FINE Controller (FINEC) is the heart of the FINE node. It handles the allocation and deallocation of resources and the overall local logic of the service.

Each FINEC can be deployed in some of the three modes independent (server), dependent (client) and autonomous (agent) — with respect to the user/network configuration, thus allowing a dynamically configurable (per user/ per node/ per service) centralised or distributed service architecture.

Services are implemented in the FINEC by true Flexible Service Logic (FSL) components interfacing to their subparts, micro service logic ( $\mu \mathrm{SL}, \mu$-services), and resources in the resource platforms (RP) directly or via interfaces to other FINEs in the network according to the 'multiple SCP-IP' model (Service Control Point-Intelligent Peripheral), cf. Fig. 3.

\subsection{Resource Platforms}

The Resource Platforms (RPs) are dedicated IPs that implement media delivery and retrieval — reception, storage and response — , as well as Specialized Resource Functions (SRF) such as collecting user input, playing announcements and invoking ROSE operations (e.g. media/user recognition and conversion).

Appropriate RPs are assigned to incoming and outgoing calls, as well as to service logic by the FINEC. There are three different RP types: one for offline media delivery and retrieval (e.g. unified messaging), one for on-line communication (e.g. internet telephony and conferencing) and one for service enacting via programmable Customer Service Control (CSC) interface - on-line via IVR/VR/DTMF/http/wap and off-line via PING agents. 


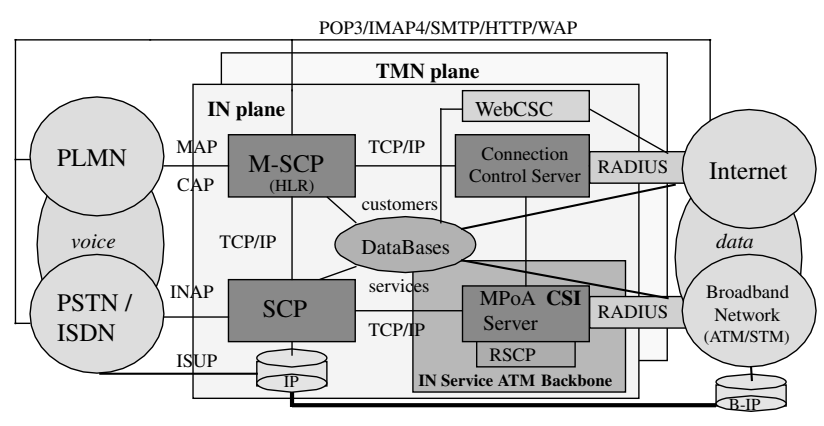

Fig. 4. A clustered SCP configuration for converged networks.

\subsection{Channel matrix switch}

The Channel Matrix Switch (CMS) is controlled by the FINEC and it routes calls from PSTN/ISDN/PLMN to the appropriate RPs via circuit call related signalling (ISUP, TCAP, MAP).

\subsection{Data Packet Switch}

The Data Packet Switch (DPS) is controlled by the FINEC and it routes calls from the internet to the appropriate $\mathrm{UMS} / \mathrm{B}$ or to the PINGA.

\subsection{Media conversion processors}

The Media Conversion Processors (MCP) have the task of converting media messages upon $\mathrm{CSC}$ request:

1. between RP-specific and external formats, and

2. between two different RP-specific formats.

\subsection{Internet gateway}

The Internet Gateway (IG) basically consists of:

- a Unified Media Bus (UMB) enabling the on-line communication between users in both network domains along dedicated lines for configuration, remote access and telephony;

- a UMS with customer disk partitions for message archivation in a Unified Media Format (UMF); and

- a PINGA for network computing by programming individual agents to fulfil specific tasks such as "wake-up" calls, news delivery or database access. It is connected to the UMS and via the UMB to the resource platforms.

By default, each UMS mailbox functions as an answering machine for the corresponding customer access line (Universal Personal Number, UPN). The PING agents are called, activated and programmed also through the mailbox using a Web/IVR/VR/DTMF interface. They are designed (Service Creation), configured (CSC) and used (Service Deployment) by the customer herself, e.g. by using scripting technology to realise definite information services. The
PINGA paradigm addresses an active mailbox in the FINE. In addition, the internet data access router (RP) has a newsgroup agent for threads selected via the CSC menu.

\section{The collision of the intelligence paradigms}

There is a definite distribution of functions and roles among network components within outbound-signalling telephony based IN. Thus, the standard SCP-IP relationship is e.g. not simply the client-server one (typical for data networks), but rather a processor-coprocessor delegation of service logic (e.g. playing announcements and collecting user input) to a subordinate unit, the Intelligent Peripheral, which delivers back some result to the SCP. The SCP then continues to control the flow of the service in the wellknown main() routine manner. Even in a distributed IN, Service Nodes are not supposed to serve each other in the common "data packet" sense, but rather to have a local (centralised) control over certain resources, services and subscribers. In addition, ongoing IN standardisation do not consider alternative network technology integration paradigms, but rather extend the SS7 model by ROSE functionality, such as e.g. invoking functions (cf. ETSI INAP CS-2).

On the other hand, the packet data world is handling intelligence not in a callflow but rather in an application oriented way, while addressing variable bandwidth technology and OSI protocol stack permutations such as e.g. running SS7 over TCP/IP ([6]).

Such developments are a clear sign that the layered architectures and the fixed treatment of IN functions, databases and services can no longer exist in a heterogeneous distributed network under rapid development, where even sculptured object paradigms such as CORBA, TINA, or DCOM and powerful IN architectures based on "clustered, multiprotocol SCPs" (Fig. 4) cannot handle all aspects of the network evolution as far as they remain rigid. One major aspect of the network has been neglected until now: it is temporal and living, i.e. changing. For example, proprietary IPs can grow up to autonomous multiprotocol "virus-like" CTI point solutions hiding micro data networks in themselves.

A time for change in network design thinking has come. Now, we have the chance to adopt a stepwise approach for building the kernel of a new communications cell. Where could we start from? Perhaps flexible nomadic intelligence may be an option.

In the following, flexible intelligent network elements are shown to be useful for functionally integrated multimode logic control in advanced nomadic intelligence architectures, which offer completely new perspectives for communication services in converged networks.

\section{The FINE architecture}

The fine architecture (Fig. 5) represents a self-configurable, adaptable, and domain-aware intelligence architecture, 


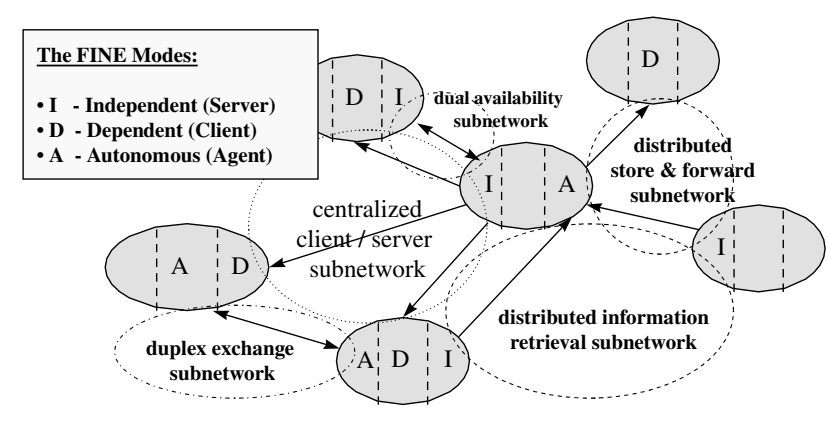

Fig. 5. An example of a FINE configuration.

both in terms of software and hardware, where network properties such as database and feature mobility are parts of the service itself. Although the FINE approach has an IN telephony origin, it can be used to virtually model any type of network.

Definition 1: A FINE (Fig. 5) is a configurable multimode network element. It can be deployed in a single, dual or triple mode of operation as independent (I), dependent (D) and autonomous (A) unit, i.e. as server, client or agent. All three modes can be deployed at the same time as far as this does not conflict with basic network design principles. A FINE may be a physical entity, a piece of software or a virtual subnetwork by itself.

Even network terminals can be regarded as FINEs operating in some of the three modes.

Definition 2: The FINE Architecture is a versatile network intelligence construction of FINEs, which are temporarily adopting certain roles in the network. It is required that:

- agents be assigned to negotiate about the distribution of roles among FINEs (active network); and

- data, functions (service logic) and roles (modes) migrate upon request from one FINE node to another, thus allowing for dynamic network configuration.

This simple model offers a unified, structured and evolutionary approach to intelligent network design and configuration, which addresses the two basic architectures: centralized and distributed IN. It allows for a flexible, but

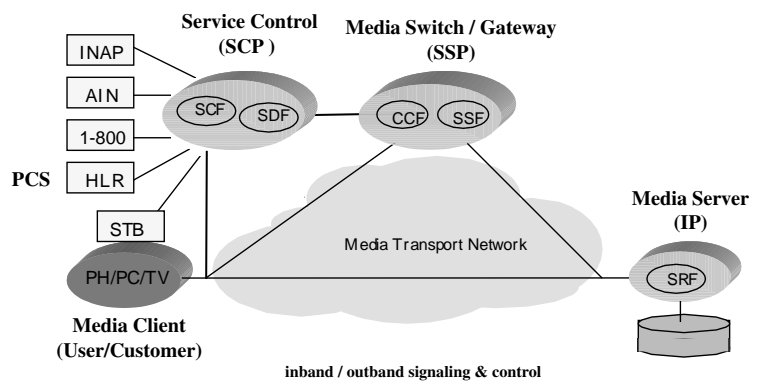

Fig. 6. Centralised FINE architecture. deterministic (event-driven) spread of intelligence across the network, matching user demands wherever and whenever required.

This approach allows the effective treatment of problems such as feature interaction (multiprotocol capability), service mobility (CAMEL + ), database updates etc.

The FINE model can be easily implemented using approaches such as JTAPI [4] for applications and ATP (Agent Transfer Protocol) [5] for transferring service logic as mobile agents between networked computers.

The main advantage of this approach is that it allows sophisticated network growth, adaptation and rapid introduction of new services by making only minor changes in the available infrastructure. In this way, the FINE architecture represents a vivid object-oriented model for intelligent service provisioning and control when compared to the traditional horizontal and vertical OSI-like layered IN architecture.

In the following, I use two examples taken from [3] to illustrate the applicability of the new model.

\subsection{Example A: centralised FINE architecture}

Let us have a centralised loop video-on-demand service architecture (Fig. 6), where media contents are transferred from a Central Media Server (CMS) to terminals throughout the network. Signalling and control are performed centrally (inbound or outbound) using the SCP-IP dialogue scheme. Media transfer is performed in a continuous synchronous channel mode or in a discontinuous asynchronous buffering mode. The CMS system provides media transfer, control and management from a single site. If the system fails or becomes incapable to support connections, connections are dropped unless there is a backup CMS.

Here the FINE architecture can be configured to represent a centralised IN architecture. It requires three network elements: a media server (I mode), a media terminal client (D mode) and a control point server/client (I/D modes).

A high availability architecture as a second SCP and additional CMS can be modelled by introducing two server/client/agent (I/D/A modes) and two server/agent (I/A modes) elements instead of the single server/client and the single server, respectively.

\subsection{Example B: distributed FINE architecture}

Let us now have the same video-on-demand service as a distributed multi-server architecture that spreads out the CMS functionality throughout the network, using the concept of Local Media Server (LMS).

This configuration saves communication costs and increases system reliability at the expense of local storage capacity by allowing subscribers to access meda directly via Local Distribution Switches (LDS).

The central servers are located at the Media Information Provider (MIP) sites (Fig. 7).

Each LMS may be a mini-CMS and its media contents 


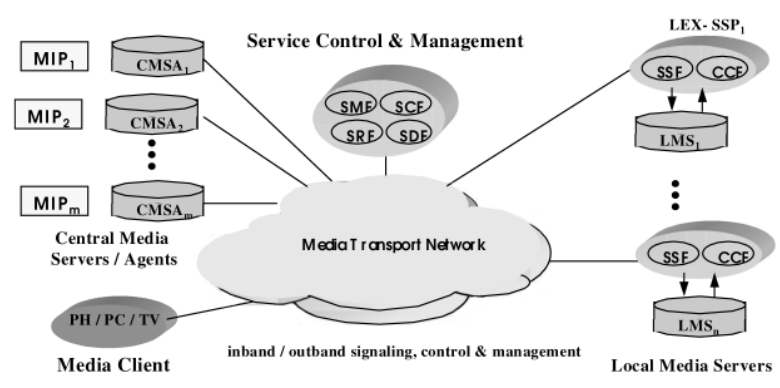

Fig. 7. Distributed FINE architecture.

may be downloaded off-line from some CVS and updated periodically.

Here, FINE may be configured in several variants from a cluster of purely centralised sub-networks of $m$ CMS sharing $n$ LMS to a cluster of purely distributed sub-network of $n$ LMS.

In a distributed sub-network, an LMS can be represented as a server (I mode) and a media terminal as a client (D mode). Modelling high availability is similar to the centralised case.

Furthermore, a single FINE may become an agent (A mode) to match SCP/SMP functionality by identifying LMS locations within distributed sub-networks and managing the overall service within the macro-network.

Of course, this function can be also cloned into a cluster of managing agents, one for each sub-network. Then, there is no reason for not keeping this one and other options free for choice, and configure the network on demand.

\section{Nomadic services and their logic}

The idea of migrating service functionality comes out of traditional network convergence solutions such as the One Number Service (ONS) for fixed and mobile networks. Figs. 8 and 9 illustrate two possible variants to realise this service architecture. The essential difference between them is the location of the Service Resource Function (SRF). We can simply ask what would happen if we let this function roam

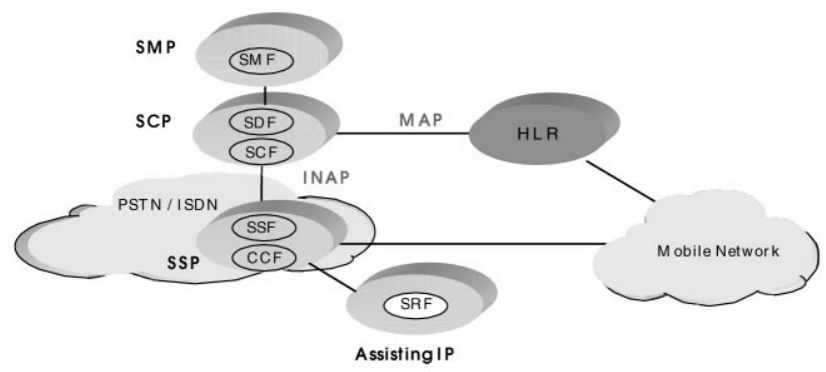

Fig. 8. One number service for PLMN and PSTN based on Assisting IP (with the kindly permission of Dr R. Rieken, Siemens AG: IN'97 Workshop (IEEE) contribution, Colorado, May 1997).

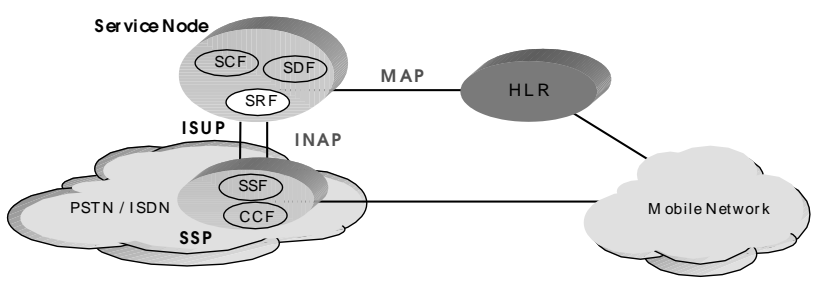

Fig. 9. One number service for PLMN and PSTN based on service node with Integrated SRF (with the kindly permission of Dr R. Rieken, Siemens AG: IN'97 Workshop (IEEE) contribution, Colorado, May 1997).

between the network elements for the reasons of evolution, effective resource utilisation, adaptability and QoS guarantee.

To assist new services and customers, intelligent networks require permanent upgrades in hardware and software components, which are not always optimally exploited at their location (Fig. 10). Even a distributed architecture, which dedicates certain resources for local usage, is not adapting (not yet) to variable load and service usage.

Thus, next to $H O W$, the question of WHERE to physically implement an IN function has always been crucial for network designers to provide for more efficient and effective services. Now, in the era of platform independent OO technology this subject obtains a new thread: mobility.

Therefore, we still have two choices:

- a non-deterministic worst-case-dimensioned service within a rigid network architecture requiring evolutionary software and hardware upgrades, or

- a deterministic service within a dynamically configurable network architecture, FINE, based on autonomous nomadic services taking care for software updates and optimal utilisation of hardware resources to a certain grade that can be proved by algorithms.

In some cases, it seems reasonable to temporarily enact and allocate/move different services and their resource areas depending on the eventual customer demands, instead of permanently increasing the functionality and the capacity of the network elements (Fig. 11).

This implies an event-driven, i.e. deterministic redistribution of roles among the network elements (i.p., client, server and agent) to satisfy network performance and user demands on configuration and QoS (cf. Fig. 12).

Definition 3: Nomadic FSLs (flexible service logic) are the smallest autonomous service elements known as

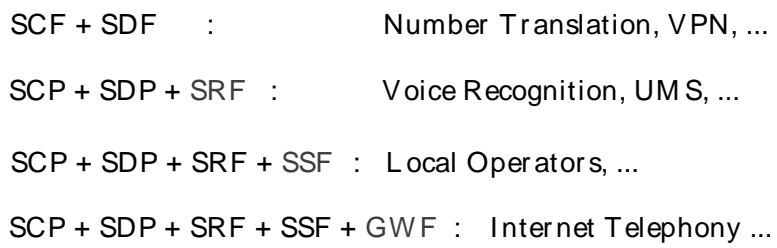

Fig. 10. Overall trend - increasing enhancement of IN functions. 


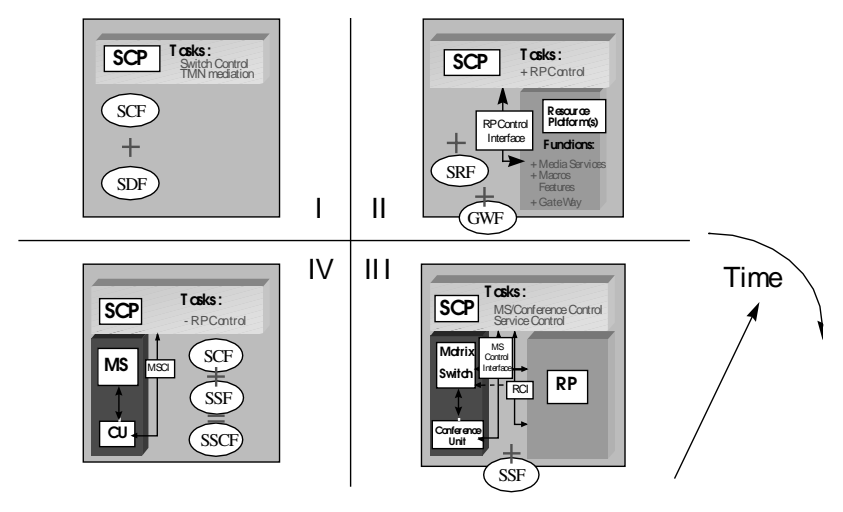

Fig. 11. A possible FINE node utilization cycle.

features (functions), including parameters (variables) and database contents being transferable from one (fixed or mobile) node of the network to another to offer intelligence on demand.

Thus, the FINE model leads to an adaptable, learning, selfconfigurable service intelligence architecture, where the database and feature mobility are parts of the service itself.

Although the FINE approach has an IN telephony origin, it can be used to virtually (re-)configure in a dynamic manner any type of network.

\section{The wandering logic of intelligence (WLI)}

Recent research on active networks approaches the problem of effective service provisioning not by moving services along network nodes, but rather by using the means of (mainly Java driven) object-oriented encapsulated software technology up to the deepest layers and elements of network protocols. No matter of whether we talk about a Netscript to program functions for sophisticated packet stream processing in network nodes [7], evolving to form capsules or datagrams carrying code [8], sprokets or "smart packets" [9], switchlets or "active packets" [10], CANEs or "composable active network elements" [11], active plugins [12] — in all these works the search for data and process customisation beyond the limits of performance, up to the

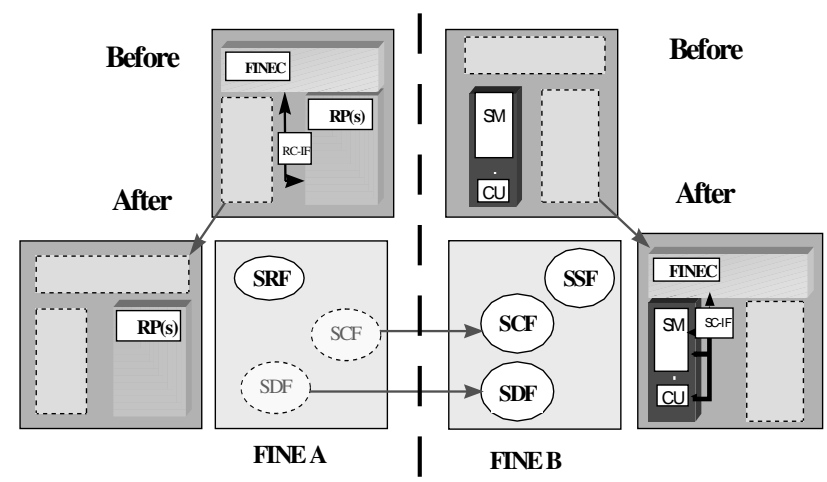

Fig. 12. A FINE reconfiguration via function split, migration and clustering. application, up to the user needs is so evident, as it was with the definition of different QoS layers matching the OSIcentric communications model a few years ago. The belief creates the reality. This holds until the next change of the paradigm. Yet, we still miss the quantum jump, the profound networking uncertainty equation.

For the time being, there are basically two approaches to improve an application's performance: service oriented and network oriented, both considered as objects, both strictly separated in hardware and software.

Programming is mainly a soft method that leaves certain options and degrees of freedom to adjust a model's behaviour towards a desired result.

If a high-level abstraction program performs well, it is then translated into a "lower" language and eventually split into modules and instructions that fit the hardware and thus perform better. Finally, if this works well, the entire program is being moulded into silicon to match the "clickto-switch" or "be there" requirement and perform for the best. Further improvements are only due to the physics.

Unfortunately, there is no way back...to check if the things could work the other way up.

If you have a new idea, you need a new model that most probably needs a new language to express its axioms, predicates etc. logic constructs. The trouble with modern computer science, however, is that it still lacks the freedom of the Greek philosophy era. Every problem is digitalised and reduced to a set of objects and functions (both corpuscules) that express the same formalism in the broad sense. Be it hardware or software, it is the same logic and the same programming language, no matter whether we unfold services or inject pro-active capsules into network nodes. What we miss is the analogous wave in the digital world, the sparking process that describes the quantum nature of evolving multiple realities, some kind of a free, Wandering Logic of Intelligence that can be at least welcome by the Lamport's strong fairness formula [13]:

$$
S F(\text { World })=\left(\square \diamond\langle\text { World }\rangle_{\mathrm{f}}\right) \vee\left(\diamond \square \neg \text { Enabled }\langle\text { World }\rangle_{\mathrm{f}}\right)
$$

where $\langle\text { World }\rangle_{\mathrm{f}}=\operatorname{World} \wedge\left(\mathrm{f}^{\prime} \neq \mathrm{f}\right)$, and $\mathrm{f}$ is its state function.

\section{Conclusions}

The FINE architecture presents a new service view at IN (in ODP terms) when compared to the classical vertical and horizontal layering of intelligence in networks. It can be centralised, distributed or both at the same time depending on the network size, architecture, service configuration, performance, scheduling and QoS requirements, as well as on the subscriber and service migration flow. This paradigm can be used as a unified, vivid object-oriented IN model, where the roles of the network elements (SSP, SCP, SMP, $\mathrm{SN}$, IP, servers, routers, CPE etc.) are temporary and on demand. Within the FINE paradigm of a dynamically 
configurable intelligent network, services and their logic are gaining a new value by becoming autonomous and truly mobile entities throughout the nodes of the network, reaching even the terminals to turn them temporarily into agents, clients or servers whenever and wherever required. Thus, service logic can be transferred, installed and mounted on demand among the FINE Controllers and the terminal equipment to provide optimal resource utilisation and QoS.

The fine architecture advantages are as follows. Replacing the deterministic with:

(1) an undeterministic approach to service deployment according to Actual User Requests and Qos requirements.

Replacing the fixed distribution of roles within the IN and data networks including tunnelling (encapsulation) of communications with:

(2) a flexible distribution of roles and functions (SCF, SDF, SFR, GWF etc.); and a

(3) Dynamic Network (Re-) Configuration user request involving

- Temporary Load Distribution,

- Temporary Resource Sharing, Control \& Distribution,

- Variable Channel and Bandwidth Allocation,

- Reuse of Software and Equipment.

The FINE Architecture is still under discussion and refinement. Currently, Siemens is offering a Messaging Gateway as a front-end solution to SCP for multimedia services at the boundary to internet (INXpress 6.1). Yet, this function may eventually move to other network elements in future. Whatever has been a network capability in the past may become a system/platform function in the future or vice versa. The choice is open.

\section{References}

[1] P.L. Simeonov, Add-On IN Gateway Services for Service Node in Distributed Telecom Networks, Nr. P30308-A8720-A000-04-7618, Siemens AG, October 1996.

[2] P.L. Simeonov et al., A Smart, Node Architecture Linking Telephony with the Internet, Proceedings of the ICCC'97, Cannes, November 18-21, 1997, pp. 77-81.

[3] Wu Tsong-Ho, et al., Distributed interactive video system design and analysis, IEEE Comm. Mag. (March) (1997) 100-108.

[4] http://java.sun.com/products/jtapi/index.html.

[5] http://www.trl.ibm.co.jp/aglets/atp/atp.htm.

[6] http://www.ttiweb.com/corporate/press/ssip-pr.htm.

[7] Y. Yemini, S. daSilva, Towards Programmable Networks, IFIP/IEEE International Workshop on Distributed Systems: Operation and management, October 1996.

[8] D. Tennenhouse, et al., A survey of active network research, IEEE Commun. Mag. 35, (Jan) 1997 pp. 80-86.

[9] B. Swartz et al., Smart Packets for Active Networks, http:// www.net-tech.bbn.com/smtpkts/smart.ps.gz, January 1998.

[10] D. Alexander, W. Arbaugh, M. Hicks, P. Kakkar, A. Keromytis, J. Moore, C. Gunter, S. Nettles, J. Smith, The switchware active network architecture, IEEE Network, Special Issue on Active and Programmable Networks 12,3 (May/June) (1998) pp. 29-36.

[11] E. Zegura, CANEs: Composable Active Network Elements, Georgia Tech., http://www.cc.gatech.edu/projects/canes/.

[12] D.S. Decasper, et al., A scalable high-performance active network node, IEEE Network (Jan/Feb) (1999) 8-19.

[13] L. Lamport, The Temporal Logic of Actions. SRC Res. Report 79, DEC, Sys. Res. Center, December 1991 